\title{
AUTOMATIC CONTROL OF SHIP MOTION CONDUCTING SEARCH IN OPEN WATERS
}

\author{
Krzysztof Stefan Kula \\ Gdynia Maritime University, Poland
}

\begin{abstract}
In this work a search operation support system is presented, which is an additional autopilot function block. Its task is to lead a vessel included in the search by the SAR services to the datum position on the basis of the data entered by the operator into the system and then automatically to search the indicated area according to the newly defined search pattern. The set goal can be achieved thanks to the autopilot that guides the ship along a given trajectory consisting of straight lines and arcs. High control accuracy is provided by the IMC control system using a relatively simple non-linear ship model. The simulation tests of the tanker model confirmed that the indicated search area can be precisely checked in a shorter time than when using the expanding square search pattern.
\end{abstract}

Keywords: : computer control, IMC control, safety and risk analysis

\section{INTRODUCTION}

Oceans and seas provide convenient opportunities for the transport of people and goods. Unfortunately, ships involved in sea transport are exposed not only to typical transport accidents, most often occurring in areas where the density of shipping is high, but also due to the impact of powerful forces of nature occurring in this environment. In order to provide assistance to the injured in such cases, appropriately trained services are required. The International Convention on Maritime Search and Rescue (SAR) was adopted at an international conference held in Hamburg, Germany, in April 1979. Primarily, it was designed to improve the existing arrangements and provide a framework for carrying out search and rescue operations following accidents at sea. Many states have accepted the obligation to provide maritime SAR coordination and services on a 24-hour basis for their territories, territorial sea and where appropriate, the high seas. To fulfill these responsibilities, they established national SAR organisations or joined one or more other states to form a regional SAR organisation associated with a certain sea area.
The planning process should be carried out in accordance with scientific criteria from both probability theory and technical aspects in order to increase its effectiveness. SAR operations planned in order to detect a missing object at sea in the minimum possible time are nowadays supported by computational methods. The science of search theory was described by Koopman [13, 14] and Stone [24]. Mathematical models based on this theory were formulated by Frost for this purpose using tidal current prediction and leeway simulations [11]. The first computer-based SAR planning system was developed by the United States Coast Guard [12]. Visualisation of ocean drift models was introduced as a helpful tool in preparing actions by the SAR coordinator [7, 30]. Li [18] and Azofra et al. [2] solved the problem of optimising the deployment of rescue units so that they could cover the largest possible area of coverage while maintaining the required safety standards. Burciu [5] proposed a Bayesian Network model to assess the reliability of SAR operations. Bayesian networks, which were used in probabilistic inference concerning the causes of maritime accidents [3], were also used to determine the reliability of the search and rescue 
operations (SAR) in coordination centres [22]. A simulation model to evaluate the performance of a SAR system was constructed by Goerlandt et al. [6].

As is often the case with road traffic accidents, the vessels closest to the scene of the incident are fundamental to the effectiveness of the assistance provided. Under long-standing traditions of the sea and various provisions of international law, ship masters are obliged to assist others in distress at sea whenever they can safely do so. In the case of SAR operations, when the distance from the location of an event is greater than $150 \mathrm{Mm}$, units from outside the SAR resources are included in the action. The Coordination Centre recommends the involvement of the vessel in the search action and outlines the proposal for its framework. However, the final decision in this regard is taken by the master of the vessel, who has the necessary knowledge of the characteristics and condition of the ship.

Bearing this kind of SAR operation in mind, the concept of extending the autopilot function with a system that will allow for more effective participation of these units in the search action is considered in this article. The aim of this work was to develop a system whose purpose is to facilitate merchant ships (incorporated into the SAR action) in carrying out an optimal search of the designated area. This tool, referred to as a search operation support system, SOSS, is an extension of modern digital autopilots with an additional function block. Its tasks include planning a search route and automatic control of the ship motion that guides it along the set trajectory of the route determined in the first stage.

\section{COORDINATION OF SEARCH AND RESCUE OPERATION}

The SAR system in each country, besides the coordinator representing the highest level of SAR management, has a Search and Rescue Mission Coordinator (SMC) and, if necessary, on-scene coordinators. The SMC typically provides the search or rescue action plan, but may also be required to perform other operations.

The elements of the basic problem of an optimal search are defined by Koopman [13]. They are as follows:

- an a priori probability density distribution on the search object location, with which it is possible to estimate the probability of containment (POC) for any subset of the possible area,

- a detection function relating to the search effort density or coverage $\mathrm{C}$ and the probability of detecting (POD) the object if it is in a searched area,

- maximising the probability of finding the object, often referred to as the probability of success (POS), which is subject to constraints on the effort involved.

In the case of searching for survivors, the data provided by the SMC contains a brief description of the incident and a recommended search area. It includes primarily the centre point, $R_{o}$ - the radius of the circle in which it is located, and sometimes size corner points. The region of the incident will usually be divided into certain sectors, inside each of which the POD is constant, though their POC may be different.

The SAR handbook [17] recommends the use of expanding square search, sector search track, line search or parallel sweep search in an open sea.

\section{DIRECT SEARCH ACTION}

Based on information from the SMC, including estimation of the most probable position of a distressed craft or survivors and determination of the search area, taking a drift effect into consideration, the assisting ship or, if there are more, the on-scene coordinator, can choose the search pattern and develop a search plan. The SAR headquarters have adopted certain rules of search action planning. They are largely based on the theory presented in Koopman's works. Determination of the optimal route of search requires consideration of the state of the sea, the time of day and weather conditions as well as steering of the ship. The classical search planning method (CSPM) assumes that the detection (vs. coverage) function is based upon the inverse cube law of visual detection:

$$
\gamma \approx k h / r^{3}
$$

where $k$ - constant of proportionality, $r$ - lateral range from the observer to the search object, $h$ - height above the ocean's surface, being applied under uniform search conditions by using search patterns consisting of long, straight, equally spaced, parallel tracks relative to the search object.

This method also assumes that the optimal search area is an area centred on the datum that is searched with uniform density of the search effort.

\section{EXPANDING SQUARE SEARCH}

Most search patterns consist of parallel tracks covering a rectangular area. Non-SAR units near the scene of the accident usually use the so-called expanding square method for their search operations. The shape of the search route recommended by SAR is shown in Fig. 1.

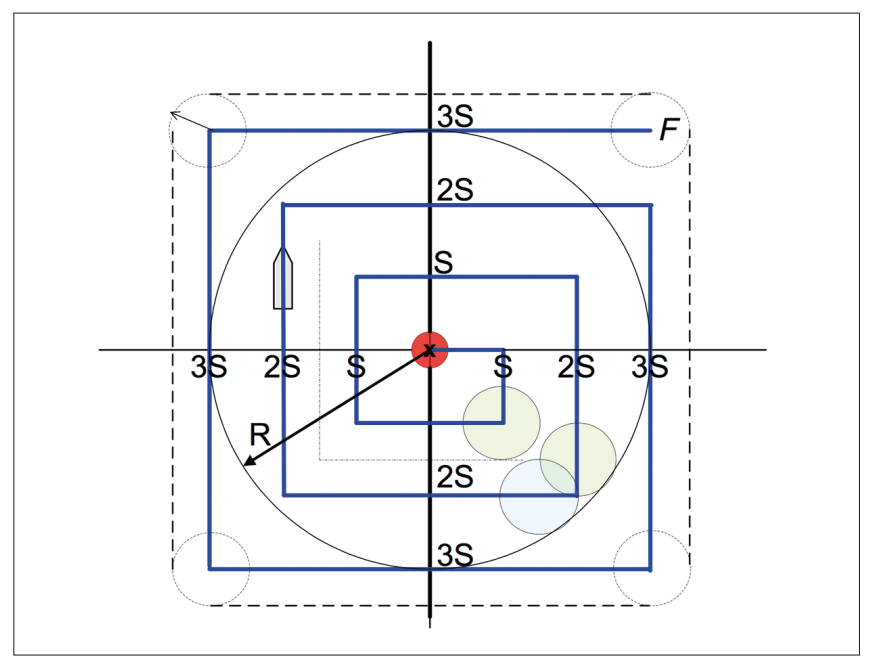

Fig. 1. Expanding square search pattern 
The commence search point CSP is specified by the SMC. Usually it is the most likely location of the missing objects. The recommended route consists of pairs of segments running perpendicularly to each other, whose length gradually increases by a segment equal to the sweep band width. The distance between adjacent tracks is called the track spacing $S$.

Suppose that we are searching uniformly throughout a region of area $A_{s}$ with the effectiveness of "random" search. The ship is moving at speed $V$ and its sweep width is W. A certain measure of the effectiveness of the sweeping process is called, in SAR terminology, coverage $C$, which expresses the density of the search effort in the region:

$$
C=Z / A_{S}
$$

where $Z$ - area effectively swept.

This may be determined by multiplying the effective search width and the effort defined as the total length of the searchers' tracks while searching:

$$
Z=W \cdot L
$$

The effective search width, if a searcher passes through a swarm of identical stationary objects uniformly distributed over a large area, is defined by the equation [12]

$$
W=\frac{\Sigma O /[t]}{(\Sigma O / A) \cdot V}
$$

where $\Sigma O /[t]$ number of objects detected per unit time, $\Sigma O /[A]$ number of objects per unit area, $V$ searcher speed and where all the values are averages over a statistically significant sampling period. If the lateral range function is known for a given search situation, then the area under the lateral range curve equals the sweep width $W$ for that situation. That is, if the detection probability is expressed as a function $d_{r}$ of lateral range $r$ from a sensor's single straight track through the swarm of objects,

then

$$
d_{r}(r)=1-e^{-\frac{2 m}{r^{2}}}
$$

$$
W=\int_{-\infty}^{+\infty} d r d r
$$

This effective sweep width is also twice the maximum detection range of an "equivalent" definite range detection profile (one that is $100 \%$ effective out to some definite lateral range either side of its track and completely ineffective beyond that range). Here, "equivalent" means that the definite range detection profile and the actual detection profile both detect, on average, the same number of objects per unit time under the same conditions of object density and the searcher's speed.

The total length of the route covered by the ship during the search in time $T$ is equal to

$$
L=\int_{0}^{T_{s}} V d t
$$

As we consider this search pattern in detail, it can be seen that the adopted track spacing is by no means constant.
This is the case if the course changes at a right angle as illustrated in Fig. 2.

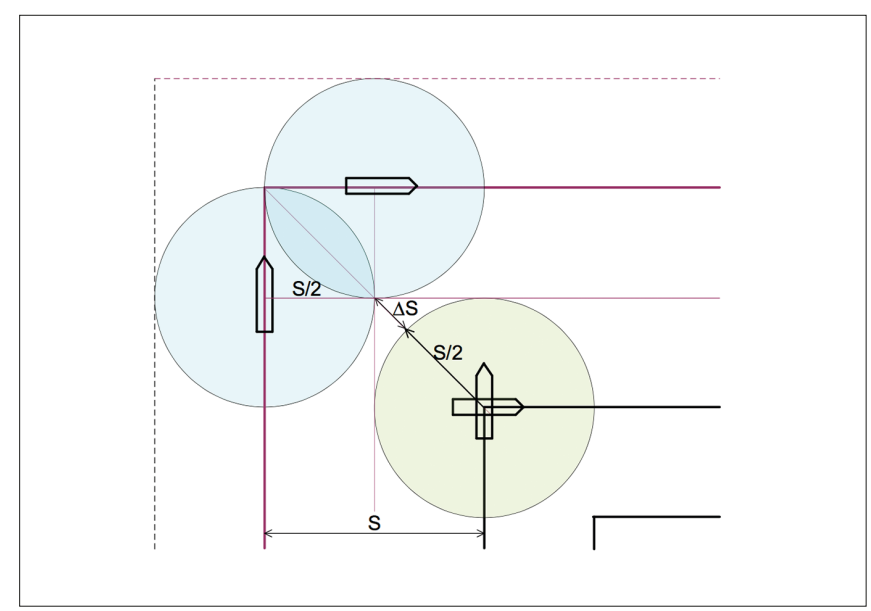

Fig. 2. Changing the sweep width in the corner of the square

As shown, there will be areas farther from the observer on the vessel than the assumed track spacing. In other words, the maximum lateral distance between the observer and the sensor (survivor) $r$ can be greater than $S / 2$ by $\Delta S$, whose value can be determined from the following expression:

$$
\Delta S=\frac{\sqrt{2} S}{2}-\frac{S}{2}=0.2071 S
$$

\section{SPIRAL SEARCH}

Of course, light and manoeuvrable SAR units can make such a return at approximately right angles. It is different in the case of large merchant ships, whose minimum turning radius is much bigger. The new search pattern proposed here takes into account the real manoeuvring properties of larger ships. Its diagram is shown in Fig. 3.

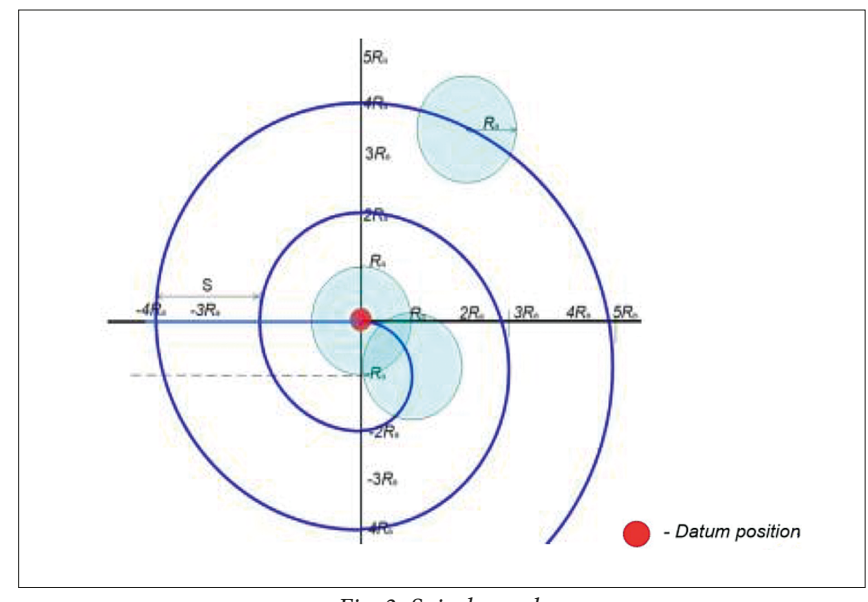

Fig. 3. Spiral search

According to this pattern, apart from the immediate neighbourhood of commence point search which avoids duplication of the searched area, the ship sails in a circle with a radius increasing after a 180-degree change of course. The planned trajectory, therefore, consists of half-circles with a radius equal to the multiple of track spacing. 


\section{COMPARISON OF TWO SEARCH METHODS}

In order to compare this method with the expanding square search pattern, the length of the route $L$ the ship has to travel to search the area indicated by the SMC and the time $t_{s}$ needed to complete it were considered.

Let's assume that the distance sailed by a ship using the expanding square search pattern is the sum of straight sections, with adjacent sections having the same length, then

$$
L_{s q}=2 \sum_{i=1}^{n} i \cdot S
$$

where $n \cdot S$ - length of the side of the largest square.

Referring to the expression for the sum of the $n$-first elements of the partial sum of this series, we will obtain

$$
\sum_{i=1}^{n} i \cdot S=S \frac{n(n+1)}{2}
$$

As the next two consecutive straight sections are of the same length, the whole length of the route will be double, so

$$
L_{s q}=n(n+1) S
$$

In order for the ship to close the square and reach e.g. position $F$ (Fig. 1), she must additionally travel a distance equal to the length of the last side, i.e. $n \cdot S$. Then, the total distance travelled $L_{t}$ while sweeping the square is

$$
L_{t s q}=n(n+2) S
$$

In the case of the new search pattern considered, the distance sailed by the vessel will be the sum of half-circles with an increasing radius value. The length of half-circles with equal radii is equal to $0.5 \pi S, \pi S$, and $1.5 \pi S$, respectively. Therefore, the total distance travelled by the vessel can be determined on the basis of the following formula:

$$
L_{s p}=0.5 \pi S \sum_{i=1}^{n} i
$$

where $n$ - number of the largest half-circle.

Taking into account the expression for the partial sum of natural numbers, we will receive the expression for the ship route consisting of semicircles:

$$
L_{s p}=0.25 \pi n(n+1) S
$$

Considering that the search area for vessels does not normally exceed $20 \mathrm{NM}$, we compare in Table 1 the $f$-indices only from a small range. The area to be searched is given by the SMC in the form of a circle with a radius $R_{o}$ around the datum. In the expanding square method, this circle is to be inscribed in the largest square, thus its side $n S$ will be equal to $2 R_{o}$. Assuming that it is a multiple of the track spacing, in the expanding square pattern the ship will pass the route

$$
L_{t s q}=2(n+2) R_{o}
$$

In the case of the spiral method, taking into account the dependence $S=2 R_{o} / n$ and the closing of the last circle, the total route can be computed from the expression

$$
L_{t s p}=\pi(n+2) R_{o} / 2
$$

Tab. 1. Comparison of lengths of routes needed to sweep through a given area with a radius $R_{0}$

\begin{tabular}{|c|c|c|c|c|c|}
\hline$n$ & 3 & 4 & 5 & 6 & 8 \\
\hline$L_{t s q}$ & $10 R_{o}$ & $12 R_{o}$ & $14 R_{o}$ & $16 R_{o}$ & $20 R_{o}$ \\
\hline$L_{t s p}$ & $7.85 R_{o}$ & $9.42 R_{o}$ & $11 R_{o}$ & 12.57 & $15.7 R_{o}$ \\
\hline
\end{tabular}

As can be seen from Table 1, the amount of search effort required to cover the area recommended by the Coordination Centre using the expanding square pattern is greater. However, it should be taken into account that, after crossing the $L_{t s q}$ route, the swept area, although partly outside the area indicated by the SAR services, is in fact $21.5 \%$ larger than it.

The second comparison criterion is the time $t s$ needed to cover the area indicated by the SAR services, assuming the same propulsion of the vessel. Since the average speed of the vessel, which depends on the rudder angle and changes in time, plays a role in this criterion, the comparison of the two methods was carried out on the basis of simulation tests, which are described in one of the next chapters.

\section{PROBABILITY OF SUCCESS}

The probability of success can be computed as $\mathrm{POC} \times \mathrm{POD}$. If the goal is to maximise it, this creates new opportunities when planning a rescue operation, but to compare the two methods, it is enough only to examine the POD vs. the effort density detection function. It should be noted that the following dependences were derived assuming that, in order to conduct search operations in an area, it is necessary to move the sensor along a series of long, straight, equally spaced parallel tracks. Koopman derived the general POD vs. the coverage detection function for this type of search using the simplified inverse cube model of visual detection and found [13] that

$$
P O D=\operatorname{erf}\left(\frac{\sqrt{\pi} W}{2 S}\right)
$$

where erf is the error function

$$
\operatorname{erf}(r)=\frac{2}{\sqrt{\pi}} \int_{0}^{r} e^{-t^{2}} d t
$$

The classical search planning method assumes that, for all searches, the track spacing will equal the sweep width, i.e. the coverage will be 1.0. If parallel tracks occur during searches (Fig. $4 \mathrm{~b}$ ), then the probability of detection will drop halfway between them and then increase again. If the tracks are spaced one sweep width apart, the average cumulative POD should be about 79\%. Let's also consider the impact on the POD that the lateral range in the expanding square pattern can have. Although the conditions of observation in search 
square corners are slightly different from those of parallel bands, there are some areas that are more distant from the observer than half the track spacing distance. The increase in the distance between two paths in this search area is shown below in Fig. 4a, where the length of the route travelled by the ship is plotted on the horizontal axis.

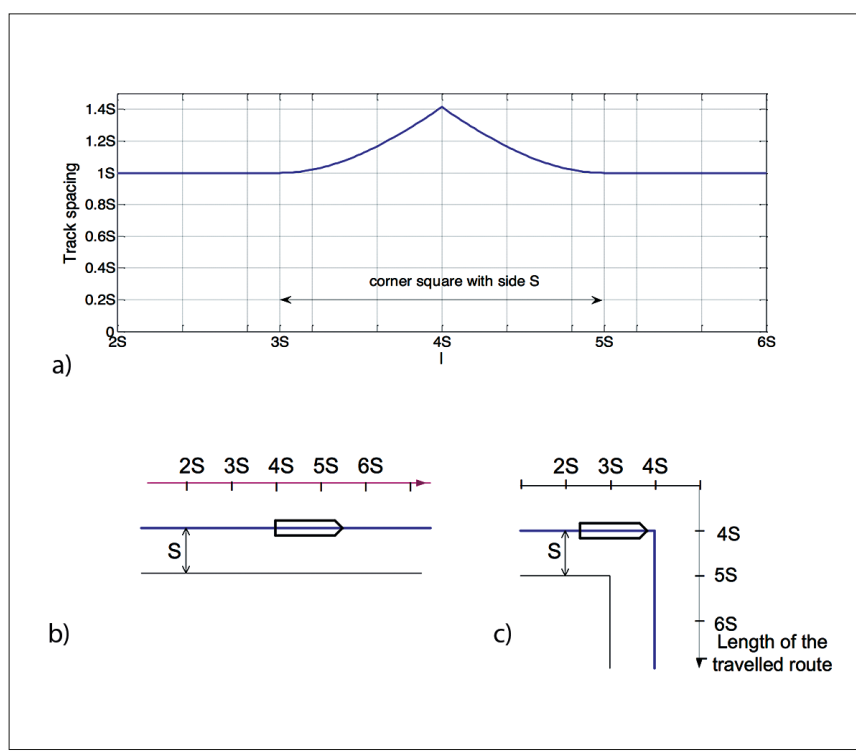

Fig. 4. a) Real track spacing varying along the side of the square, b) Parallel tracks, c) Square search

The lateral range near the vertices of each square increases by $\Delta S$ (8) regardless of the ship's turning radius, as shown in Fig. 2.

Suppose that track spacing $S=W$. On the basis of the above dependences (17), we get that the POD $=0.7899$. However, if the lateral range from the observer to the sensor increases to half the maximum distance between adjacent routes and reaches the value of $1.207 \cdot \mathrm{W}$, the probability of detection decreases to a value of 0.7009 . The probability that the distance between the observer and the missing object near the vertices of the square will be greater than $0.5 \mathrm{~S}$ is due to the ratio of the area, in which this condition is met for the total surface of the sweep band

$$
\mathrm{P}(\mathrm{r}>0.5 S)=\frac{4 S^{2}}{[2 \mathrm{nS}+2(\mathrm{n}-2) S] S}
$$

This can be written as

$$
\mathrm{P}(\mathrm{r}>0.5 S)=\frac{1}{\mathrm{n}-1}
$$

which means that the longer the sweep bands are, the smaller will be the drop of the POD caused by a temporary increase in the distance between tracks.

The average value of the range extension in corner squares of the sweep band is

$$
m=\frac{1}{2 S} \int_{i S}^{(i+2) S} \delta d l=1.1478 S
$$

Fig. 5 shows the graphical dependence of the POD on the search effort density, which is expressed here as the ratio $W / S$.

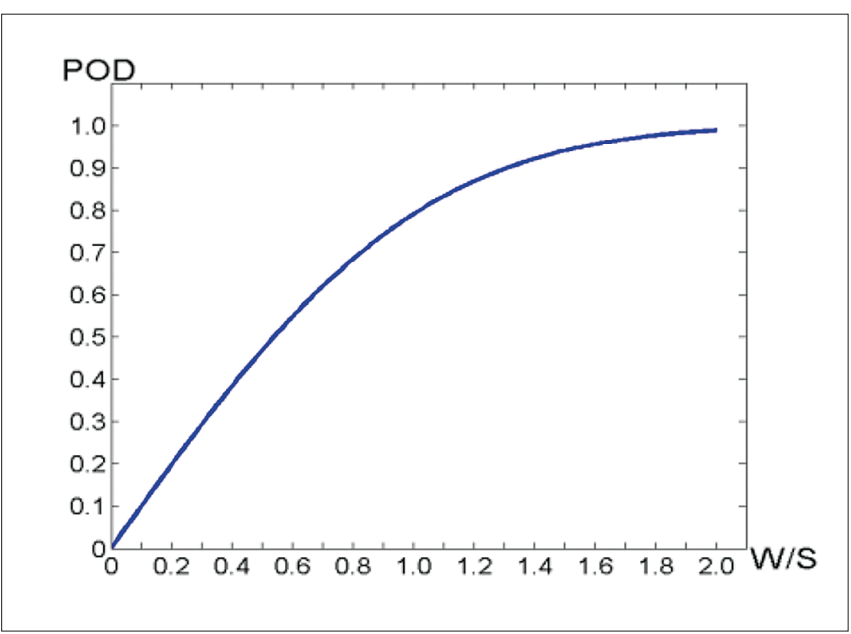

Fig. 5. POD dependence on coverage

For the coverage equal to unity, the POD is $79 \%$. If the distance between adjacent routes is in the extreme case equal to $1.207 \mathrm{~W}$, the coverage falls to the value of 0.83 and the POD to $70 \%$.

\section{DURATION OF THE SEARCH}

The second comparison criterion is the time $t_{s}$ needed to cover the area indicated by the SAR services, assuming the same propulsion of the vessel.

When planning a search, the objective is often to maximise the probability of detecting the missing object by some time. Because the survival time of a human without special protective clothing rapidly reduces as the water temperature decreases, a special case of SAR action is to maximise the probability of detecting the survivor alive.

If we are searching uniformly throughout a region of area $A$ with the effectiveness of "random" search, then the probability of detecting the search object by time $t$ can be expressed by the so-called random search formula

$$
P O D=1-e^{-\frac{W V(t) t}{A}}
$$

If we take into account Table 1, it follows from this dependence that the spiral method can be a useful tool for arranging a plan of the SAR action.

Determining the optimal sweep width on the basis of factors influencing its value due to their fuzziness is difficult to explain. Interpreting them has a significant impact on the risk. On the one hand, by reducing the track spacing, the probability of overlooking the missing object decreases. On the other hand, it may increase the time frame for finding the survivors. This may not matter much when they are in a well-stocked, unsinkable boat. Otherwise, when people are directly affected by the environment at a low water temperature, a change of the strategy to " $T$-optimal" maximises the probability of finding the missing object by a limited time $T$. However, a higher speed will increase $A$ but reduce the search efficiency at the same time. This could increase the probability of detecting the missing object faster. 


\section{CONTROLLING THE SHIP MOTION ALONG AN ARC}

To conduct a spiral search along the arc-shaped trajectory, a ship motion control system was designed. This innovative approach to ship control was described in [16]. This chapter presents the concept of using this system, extended by a case of turning a complete 180 degrees.

Let's assume that the considered autopilot can control the ship to a given way-point. The set course $\psi_{\text {ref }}$ is not a fixed value and depends on the current bearing to the destination point. If we assume that $G(x, y)$ and the offset from the next waypoint is at the moment $t$ in the meridian line $\Delta x_{i}(t)=x_{i}-x_{s}(t)$ and in the parallel line $\Delta y_{i}(t)=y_{i}-y_{s}(t)$, then the reference course can be determined from the dependence

$$
\psi_{\text {ref }}(t)=\frac{180}{\pi} \cdot \operatorname{arctg} \frac{\Delta y_{i}}{\Delta x_{i}}+n \cdot 180 \quad[\mathrm{deg}]
$$

where $x, y$ - the coordinates of the centre of gravity of the ship, $x_{i}, y_{i}$ - the specified coordinates for the successive turning point,

$$
\begin{aligned}
& n=0 \quad \text { if } \quad \Delta x_{i}>0 \text { and } \quad \Delta y_{i}>0 \text {, } \\
& n=1 \quad \text { if } \quad \Delta x_{i}<0 \\
& n=2 \text { if } \Delta x_{i}>0 \text { and } \Delta y_{i}<0
\end{aligned}
$$

In the proposed layout, the transition from $w p_{i}$ to $w p_{i+1}$ is to take place on an arc with a given radius $R$, as illustrated in Fig. 6.

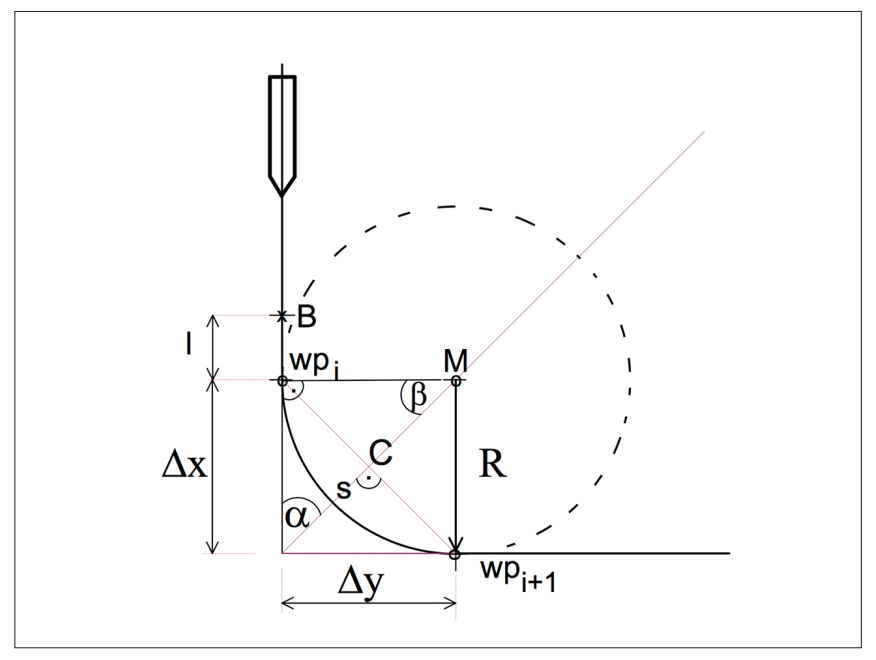

c-chord, s-sagitta, $R$-radius, $M$-middle of the circle, l-advance section, $B$-starting of the manoeuvre

Fig. 6. Turning along the set arc to a fixed way-point

The determination of a reference trajectory in the shape of an arc, which should be kept by the ship from the waypoint $w p_{i}$ to $w p_{i+1}$, requires giving their coordinates $\left(x_{i}, y_{i}\right),\left(x_{i+1}, y_{i+1}\right)$ and the ship's course $\psi_{1}, \psi_{i+1}$ at these points. This will make it possible to determine the required turning radius $R$, assuming that the turn is made by an angle of no more than 180 degrees. There is only one arc, whose slope of tangents in the points common to neighbouring straight lines will coincide with the slope of these sections. The radius of this arc can be calculated on the basis of the trigonometric dependence

$$
R=0.5 c / \cos \alpha
$$

where $\alpha \mathrm{a}$ is half of the so-called central angle corresponding to the change of the course angle by $\Delta \psi$ from $\psi_{i}$ into $\psi_{i+1}$, and $c$ means the chord, which is the length of the straight line joining the beginning and end of a desired arc and can be developed from the equation

$$
c=\sqrt{\Delta x_{i+1}^{2}+\Delta y_{i+1}^{2}}
$$

where $\Delta x_{i+1}=x_{i+1}-x_{i}$

$$
\Delta y_{i+1}=y_{i+1}-y_{i} .
$$

The angle $\alpha$ is equal to

$$
\begin{gathered}
\alpha=90^{\circ}-\beta \\
\beta=\psi_{i}-\psi_{i \rightarrow i+1}
\end{gathered}
$$

where $\psi_{i \rightarrow i+1}$ is the bearing from $w p_{i}$ to $w p_{i+1}$.

\section{CONTROL SYSTEM OF THE SHIP MOTION}

Most autohelm systems are mainly formulated to follow a desired course under constant speed settings. Because the ship, as a control plant of its motion, is highly nonlinear, the object places high demands on the regulation system. The course control is still an active field of research. Nowadays, considering structural and model parametric uncertainties in marine systems, some nonlinear adaptive techniques are being tested. In recent years, systems such as artificial neural networks $[4,23]$, sliding mode control $[9,19]$, predictive control [8, 25], fuzzy control [29], backstepping control [26] and control applying a nonlinear observer [31] have been tested to achieve high quality regulation. The advantages of the IMC for the control of the ship motion are presented in [28]. Because it is not suitable for controlling integral processes, the structure of the system has been modified to one in which the angular velocity is regulated. The IMC [20] is generally an open-loop control system, in which the input signal is corrected according to the difference between the plant and the model. The diagram of this control system is shown in Fig. 7.

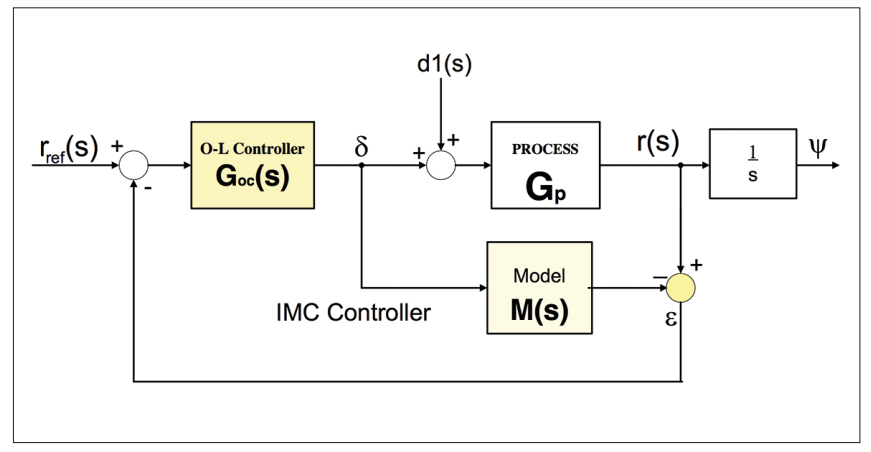

Fig. 7. Structure of Internal Model Control IMC 
The signal of mistuning of the model and the object $\varepsilon$ may be the result of the influence of disturbances, model uncertainty or model incorrectness. The error signal can adjust the set point in such a way that the value, adjustable in the steady state, is equal to the desired value, even when the model differs from the object. The transfer function of this system is equal to

$$
G(s)=\frac{r(S)}{r_{\mathrm{ref}}(S)}=\frac{G_{p}(s) G_{o c}(s)}{1-M(s) G_{o c}(s)+G_{p}(s) G_{o c}(s)}
$$

Let's assume that the dynamics of the linearised plant is presented in the form of the transfer function $G_{p}(s)$ and that the model fully reflects the properties of the object $M(s)=G_{p}(s)$. Then, the controller which could ensure the perfect control in this system should have the transfer function equal to the inverse of the object

$$
G_{o c}(s)=M_{i n v}(s)
$$

where $\mathrm{M}_{i n v}(s)$ is an inverse model of the process taking into account rules of $Q$-parametrisation.

To make this controller realisable, it is necessary to add into the inverse model the forming filter so that its transfer function should be proper

$$
Q(s)=F(s) \cdot M_{i n v}(s)
$$

where $F(s)$ - the transfer function of the filter.

\section{MODEL OF THE SHIP}

It can be seen from Eq. (28) that, in the absence of uncertainties and plant modelling errors Eq. (29), the control system works as an open-loop system. The performance of the control system will depend on the accuracy of the model. Unfortunately, the dependences describing the ship's motion are nonlinear. The movement of a ship can be described using six nonlinear differential equations [10], which are based on the equations of moments and forces acting on a ship in three dimensions. The first step to obtain a simplified model is to omit some coupling between the oscillating motions of the vessel around the axes. Then, after linearisation around a selected point of work and after elimination of the sway velocity, we get the following simplified linear differential equation:

$$
\begin{gathered}
\ddot{r}(t)+\left(\frac{1}{T_{1}}+\frac{1}{T_{2}}\right) \cdot \dot{r}(t)+\frac{1}{T_{1} T_{2}} r(t)= \\
=\frac{k}{T_{1} T_{2}} \cdot\left(T_{3} \cdot \dot{\delta}(t)+\delta(t)\right)
\end{gathered}
$$

where $\delta$ - rudder angle.

The time constants $T_{1}, T_{2}, T_{3}$ and gain $k$ depend on derivatives of hydrodynamic forces and momentums with respect to the sway and surge velocity and the yaw rate $r$. Assuming constant speed of the ship, the transfer function of the model [21] is equal to

$$
M(s)=\frac{r(s)}{\delta(s)}=\frac{k\left(1+T_{3} s\right)}{\left(T_{1} s+1\right)\left(T_{2} s+1\right)}
$$

However, the change in longitudinal and lateral velocities during the manoeuvre leads to changes in the dynamics of the ship and thus increases the incorrectness of the linear model. To prevent this, in the proposed control structure, such a model with parameters depending on propeller revolutions $n$ and the rudder deflection d was introduced. Eq. (31), after rearranging to the new form,

$$
\begin{aligned}
& \ddot{r}(t)=\frac{k(n, \delta)}{T_{1} T_{2}(n, \delta)} \cdot\left(T_{3}(n, \delta) \cdot \dot{\delta}(t)+\delta(t)\right)- \\
& -\left(\frac{1}{T_{1}(n, \delta)}+\frac{1}{T_{2}(n, \delta)}\right) \dot{r}(t)-\frac{1}{T_{1} T_{2}(n, \delta)} r(t)
\end{aligned}
$$

can be directly used to create a model, whose operator transfer function with variable parameters better reflects the nonlinear properties of the ship. A schema of this second order model is shown in Fig. 8.

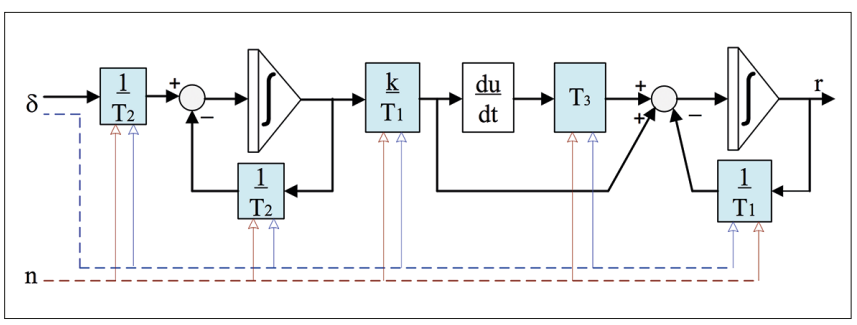

Fig. 8. Nomoto's model of the ship with variable parameters $k$ and time constants T1,T2,T3

The dashed lines indicate the influence of the abovementioned variables on the model parameters. As can be seen, deflection of the rudder is not only a control value, but also affects the value of the time constants.

The dependence of the steady state gain, time constants $T_{1}, T_{2}, T_{3}$ and the rudder angle at shaft velocity $n=200$, are shown in Fig. 9.

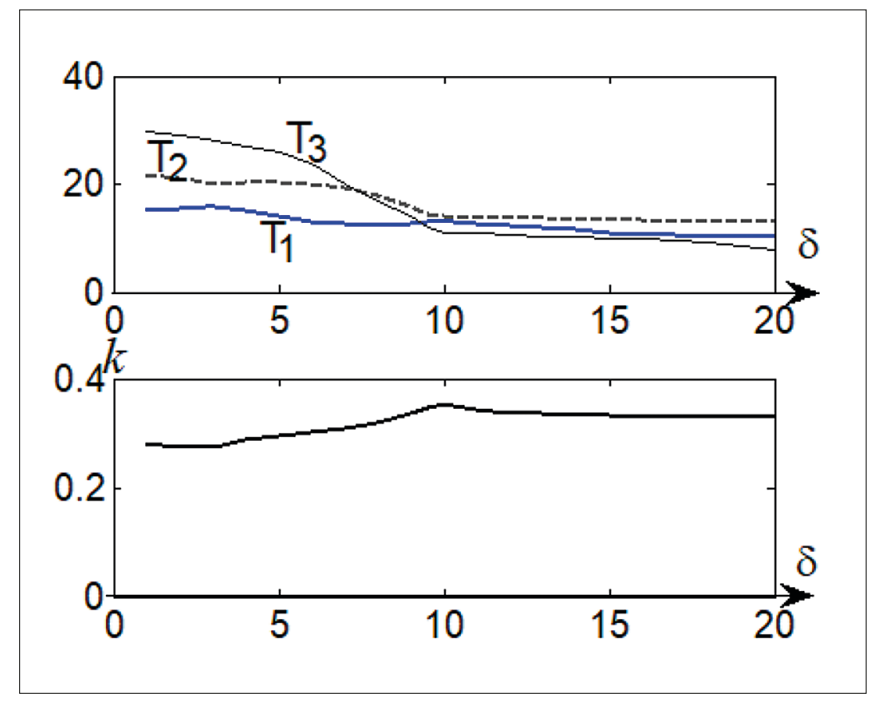

Fig. 9. Model parameters as functions of rudder deflection

The steady state gain of the model $k$ at a different number of propeller revolutions $n$ is calculated from the dependence

$$
k(\delta, n)=k(\delta, 200) \cdot n / 200
$$


This will ensure better quality of the model in a wider range of changes of the state variables of the system.

If the shaft velocity is equal to 200 revolutions per minute, then, after a 1 degree rudder deflection, the responses of the 3 DOF nonlinear model and Nomoto's model are almost the same, as presented in Fig. 10. However, with larger rudder deflections, these differences will be bigger.

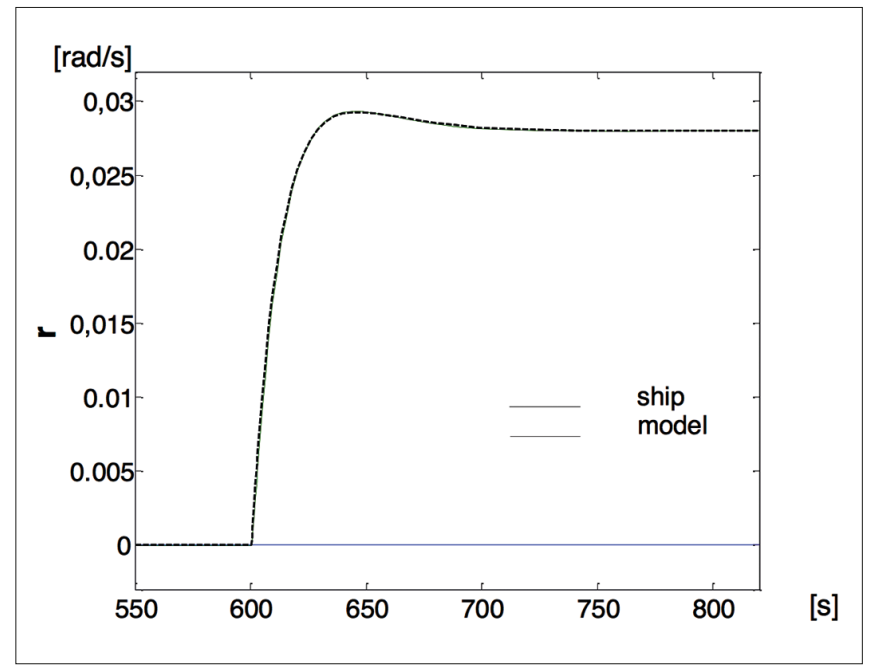

Fig. 10. Model responses of 1 deg rudder deflection $n=200, d=1(t=600), T_{1}=15 s, T_{2}=21.6 s, T_{3}=29.7, k=0.028$

In order to determine the transfer function of the IMC regulator $G_{o c}(s)$, an inverse model of the plant is required. It must be compatible with the model of the ship used, so that $M_{i n v}(s) \cdot M(s)=1$. Then the transfer function of the controller $G_{o c}(s)$ will be equal using inertia of the first order $(n=1)$ as a filter

$$
G_{o c}(s)=\frac{\left(T_{1} s+1\right)\left(T_{2} s+1\right)}{k\left(1+T_{3} s\right)\left(T_{f} s+1\right)}
$$

It should be noted at this point that such an approach as the IMC may cause arbitrary determination of the parameter $T_{f}$ to limit the system's ability to maximise the full power of the actuators. However, it is extremely useful in implementing harmonious control of nonlinear systems. The use of a linear model to control the nonlinear plant is acceptable; however, to improve the quality of control, a nonlinear model in the structure of the IMC controller could be included, causing a better reflection of the actual controlled object than a simple linear model. The advantages of such a model in controlling a nonlinear object such as a ship are presented in [15].

Such autopilots as described above cannot be directly used to automatically control a ship during spiral search. In order to be able to control the ship along the desired path consisting of straight lines and circular arcs, it is necessary to couple the control system of the ship motion with a device that, according to the designated tasks, will set the current reference value of the controlled variable. The task of this device, called the Reference Signal Builder (RSB), is to determine the set angular velocity $r_{r e f}(t)$ according to the selected operating mode such as the control to a reference course or to a chosen way-point along a straight line or an arc. The functional block schema of the RSB in the section providing control along an arc shape trajectory is shown in Fig. 11.

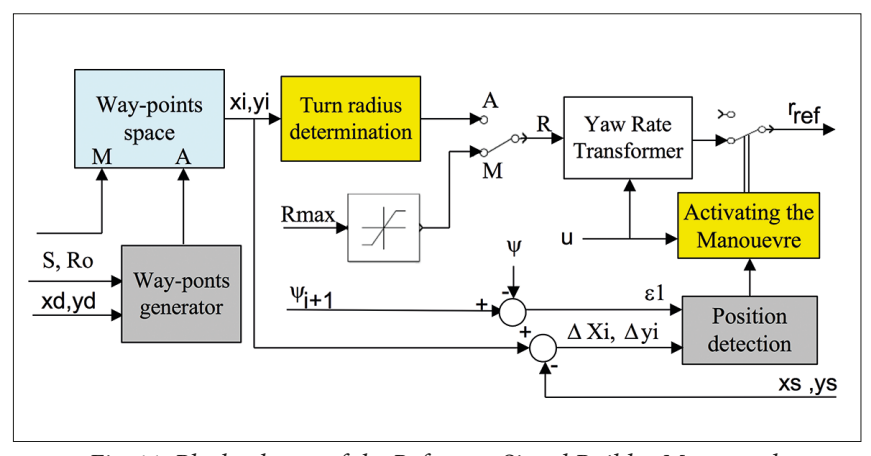

Fig. 11. Block schema of the Reference Signal Builder M-manual, A-automatic

The most common use of arc control is a course change when the curve radius is limited to the specified $R_{\max }$ (switch position M). Arc control from $w p_{i}$ to $w p_{i+1}$ requires their coordinates to be entered into the database. The set value of the turning radius $R$ is calculated in the function block "Turn radius determination" on the basis of way-points coordinates and the course to $w p_{i}$, from Eqs. (24)-(27). The yaw rate transformer sets on-line for the entire duration of the planned ship manoeuvre and the reference value of the yaw rate rref according to the measured vessel speed $u$.

In order for this system to be able to automatically search the SAR-derived area around the datum position using the spiral method, the RSB has been expanded with the waypoints generator. Before the automatic search mode can be activated, the following data: spacing $S$, acceptable deviation from consecutive way-points $\varepsilon_{2}$ and the coordinates of the commence search point $\left(x_{d}, y_{d}\right)$ as the centre of a circle with radius $R_{o}$ typed by the SMC as the most likely area to be searched, must be entered into the system. The initial value of its radius is usually taken to be $10 \mathrm{Mm}$.

Spiral search is a sequence of turns that constitute semicircles with increasing radii. Their radius at the time of transition to a new arc is determined by the dependence

$$
R_{i+1}=0.5\left(\left|x_{i+1}^{\prime}\right|+\left|x_{i}^{\prime}\right|\right) \mid
$$

where $x_{i}^{\prime}$ is the coordinate of the vertical axis, normal to the direction of approach to the starting point, which is the centre of this reference frame.

The following radius reference values are determined until the next way-point is reached. The horizontal axis $\mathrm{Y}$ of the coordinates system of the search area coincides with the direction of the ship's approach to the successive waypoints that are equal to 0 . However, in order for the entry into the spiral to actually start near the datum (starting point), the manoeuvre should start some time in advance. This is because when the rudder is turned, the ship does not immediately enter the turn. In order to increase the accuracy of the path-keeping, the rudder deflection command must precede the way-point by a certain distance $l$. The control system determines the length of advance of manoeuvre $l$, which depends on the ship's inertia based on the following dependence: 


$$
l=u(t) \cdot T(u)
$$

where $T$ - resultant time constant of Nomoto's model $T=T_{1}+T_{2}-T_{3}$, which also depends on the ship's velocity $u$.

The coordinates of the point of starting turn $B\left(x_{1}^{\prime}, y_{1}^{\prime}\right)$ that goes beyond the way-point $w p_{1}$ can be estimated as

$$
y_{1}^{\prime}=-l \quad x_{1}^{\prime}=0
$$

Accordingly, the control is redirected to this mode by the "Manoeuvre activating" block after confirming that it has reached the designated position, i.e. even before the way-point. The function block "Position detection" tracks the current position of the ship and relates it to the set target.

Transition to the next way-point occurs when one of the following conditions is met:

$$
\begin{gathered}
\varepsilon_{1}=\psi_{i+1}-\psi(t) \leq \pm 2^{\circ} \\
\varepsilon_{2}=\sqrt{\Delta x_{i}^{2}+\Delta y_{i}^{2}}<r_{t}
\end{gathered}
$$

where $r_{t}$ - distance representing an acceptable deviation from the way-point $w p_{i+1}$.

Figs. 12.a and 12.b illustrate the importance of anticipating the start of a manoeuvre. During the first, the command of the rudder was given when the ship reached $w p_{i}$ and during the second one at point $B$, a distance $l$ meters earlier $(l=11.9 \mathrm{~m})$, which allowed the vessel in the absence of disturbances to pass the planned trajectory along the arc of a circle without a shift.

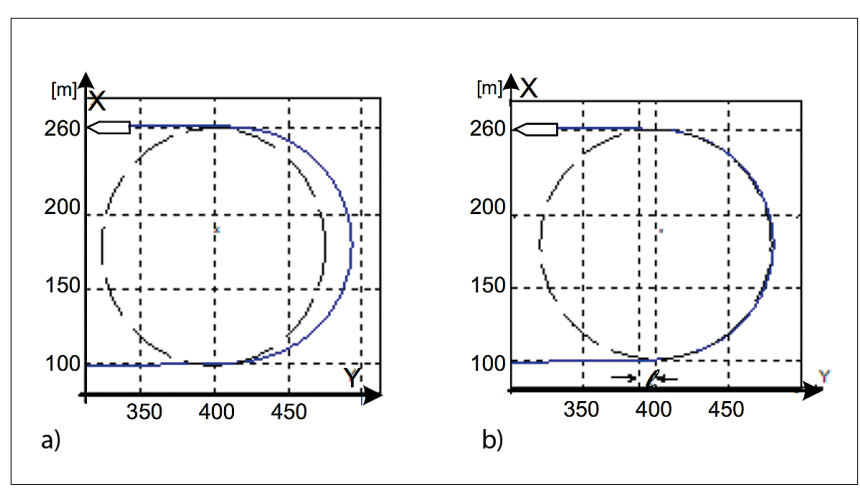

Fig. 12. Route of the ship without (a) and with (b) a shift of the manoeuvre beginning

\section{SIMULATION TESTS}

Simulation tests were carried on Lake Silm out using the model of the ship "Blue Lady" belonging to the Foundation for Safety of Navigation and Environment Protection in Iława. It provides training for masters and merchant navy officers to practise and improve their skills through trial manoeuvres in a safe environment. It is a copy of the tanker made on a scale of 1:24. For the purpose of computer simulation of the ship motion, a mathematical model verified during trials on the

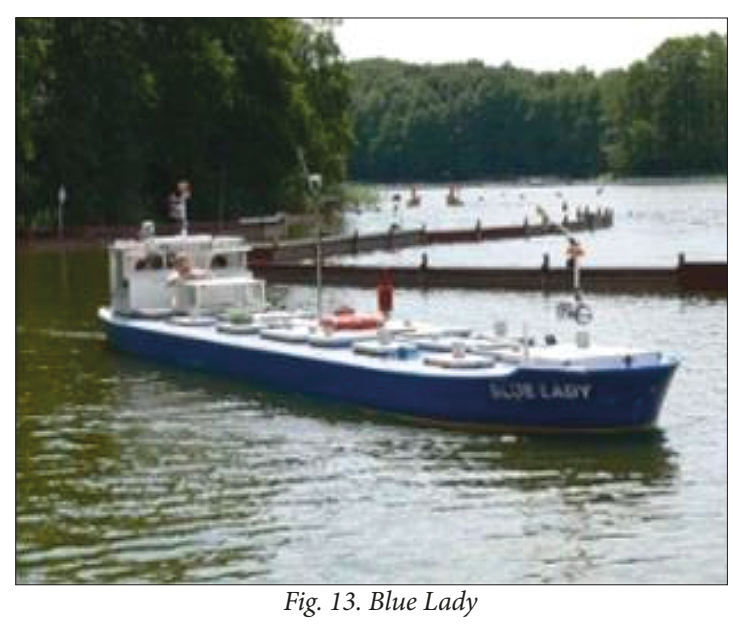

lake taken from Tomera's work [27] was used. Simulation tests were performed using the Matlab/Simulink software. The aim of the study was to verify the operation of the ship control system after the specified arc and to assess the suitability of the system to automatically perform a search of the area designated by the SAR service to rescue the survivors. For the synthesis of the IMC controller to test its robustness for model inaccuracy, a model adequate for the number of revolutions $n=200$ and rudder deflection $\delta=4$ deg was used. As the filter time constant $T_{f}$ the value of $30 \mathrm{~s}$ was adopted. Matlab's Guide User Interface GUI facilitated communication in the system (Fig. 14).

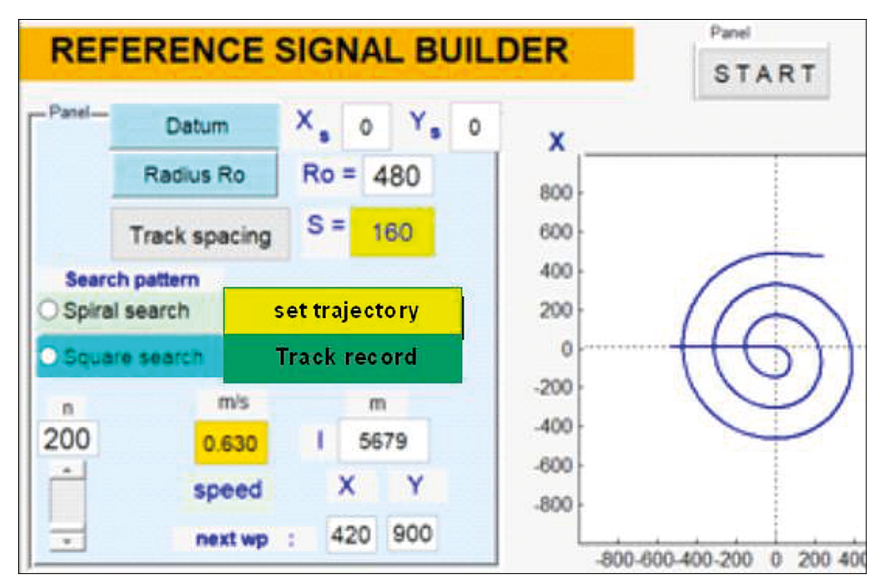

Fig. 14. Graphical User Interface GUI

Simulations of the search vessel movement according to the square search method and to the spiral search were proposed in this work. Based on the results obtained, these methods were compared in terms of the time consumed to achieve the goal. Of course, the aim is to rescue the survivors, but for the purpose of testing the methods, it was assumed that the aim would be to sweep all the points of the desired area as quickly as possible from a distance not greater than the specified effective sweep width. It was assumed that the area designated by the SAR mission coordinator to search for a small boat (less than $5 \mathrm{~m}$ ) would include a circle with a radius of $8.3 \mathrm{NM}$. The weather was windless and visibility was rated at $15 \mathrm{NM}$. After scaling, an area with radius $R_{o}=640 \mathrm{~m}$ was tested. The ship movement at full load was registered 
in a perpendicular coordinate system, where the datum position coincided with the commence search point and had the coordinates $(x=0, y=0)$. The ship speed was halfforward at the specified number of revolutions of the propeller $\mathrm{n}=200 \mathrm{rpm}$. With regard to the SAR recommendations, a track spacing value of 2.1 NM was adopted. After rescaling, this corresponded to a distance of $160 \mathrm{~m}$. In Figs. 15 and 16, the trajectory in which the vessel arrived at the starting point was shown during automatic execution of the square and spiral search. In the other method, the same turning radius was used to change the course by 90 degrees as for the first semicircle in the square method.

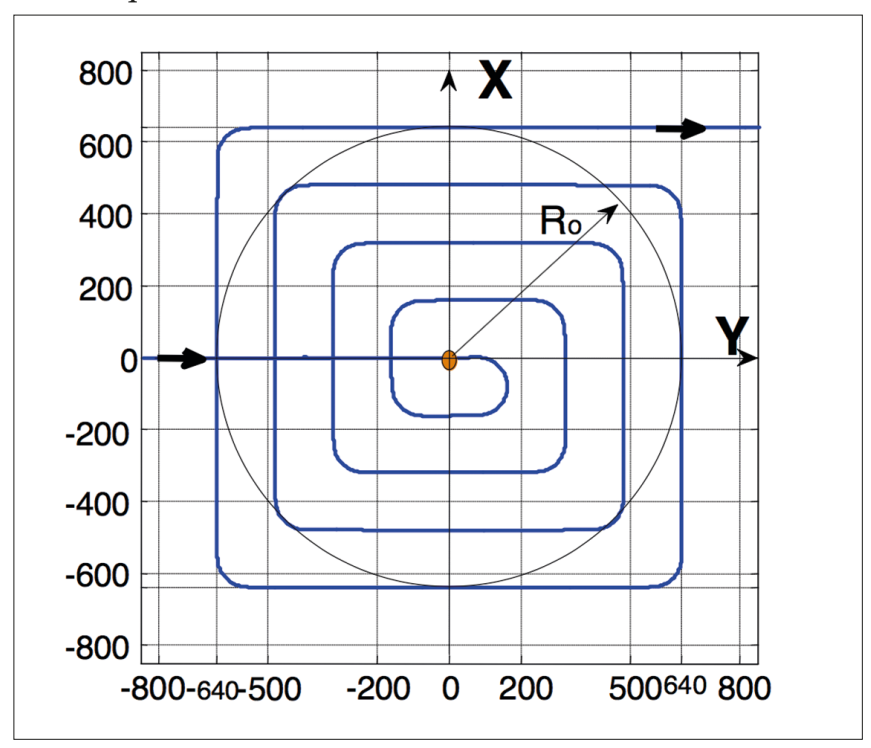

Fig. 15. Vessel trajectory during the expanded square search

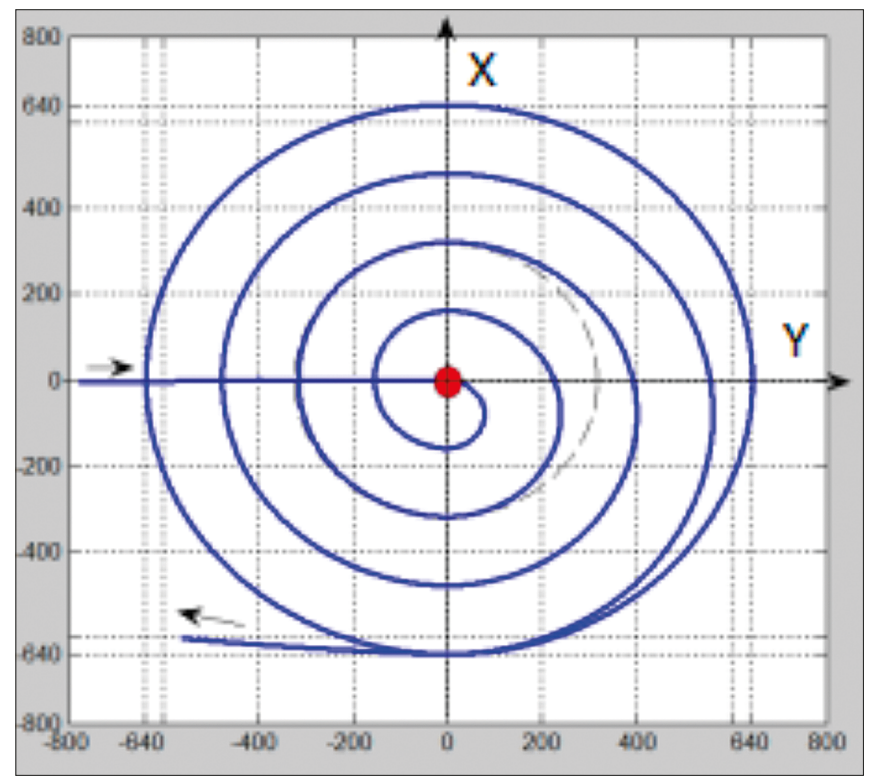

Fig. 16. Vessel trajectory during the spiral search

Performing a task using the square method requires a longer distance. According to Eq. (12), taking into account that $n=8$, the total distance $L_{t s q}$ should be equal to $80 . S$. However, 79.06 $S$ was measured, because the ship was sailing on an arc with the radius of $80 \mathrm{~m}$ when changing course.
In the spiral method, taking into account the closing of the last circle, as shown in Fig. 16, the total routes were $62.83 . \mathrm{S}$ and 64.55 .5 , respectively. Figs. 17 and 18 showed the ship's forward speed during the search. As seen in the proposed method, the hydrodynamic resistance of the hull is lower, which allows a higher average speed to be obtained when limiting the maximum speed. However, the optimal speed should in any case be determined by the shipmaster.

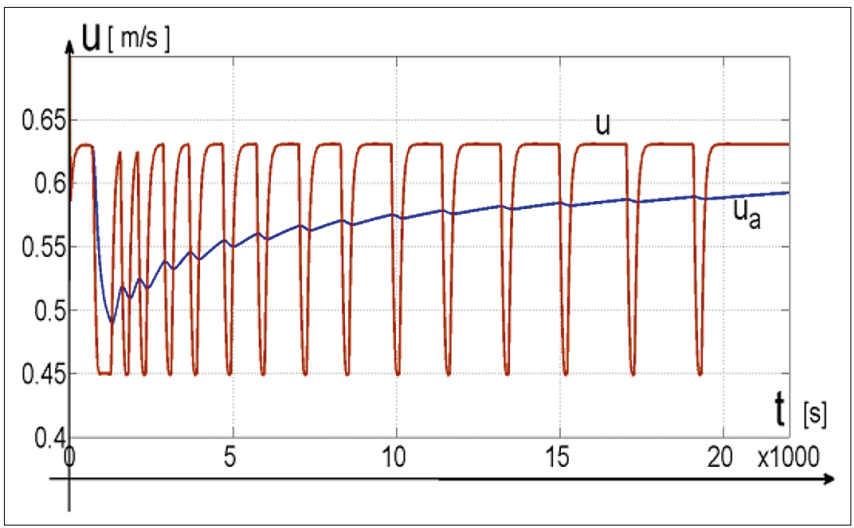

Fig. 17. Time series of vessel speed $u(t)$ and average velocity $u a(t)$ during the square search

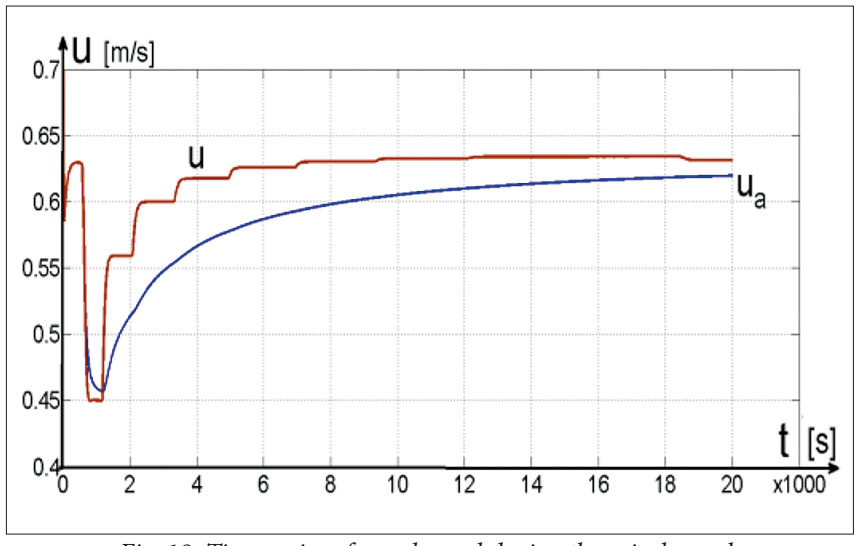

Fig. 18. Time series of vessel speed during the spiral search

Comparing the probabilities of detection of a missing object after completing the spiral test, that is, $t=19450 \mathrm{~s}$, the tested area $A_{s}$ is equal to the sum of $A_{o}$ and $A_{d}$, where $A_{d}$ doubled the search, including the closing of the last full circle and sailing to the CSP, because

$$
\begin{gathered}
A_{o}=\pi R_{o}^{2}=16 \pi S^{2} \\
A_{d}=\frac{16+7 \pi}{4} S^{2}
\end{gathered}
$$

On the basis of Eq. (22) it is possible to determine the detection probability for the tested spiral search $\mathrm{POD}_{\text {sp }}=0.6955$. In the expanded square search, after the same time, the ship reaches a point with the coordinates $(-640,142)$ and the examined surface reaches the value of 57.86. $\mathrm{S}^{2}$ and the $\mathrm{POD}_{\mathrm{sq}}=0.5951$.

Figs. 15 and 16 show the trajectories of the ship without external disturbances. However, waves, wind and sea currents may affect the ship. On the basis of the relative wind speed and wind direction, as well as the ship's response to this 
disturbance measured during the tests on the lake, a model of the wind force generator was prepared. A fragment of its recording showing changes in the relative wind speed and direction is shown in Fig. 19. The obtained wind model was then applied to simulate the ship movement [27]. The effect of wind on the ship will change the shape of the trajectory along which it will move. This depends on several factors.

First of all is whether the next half circles of the planned
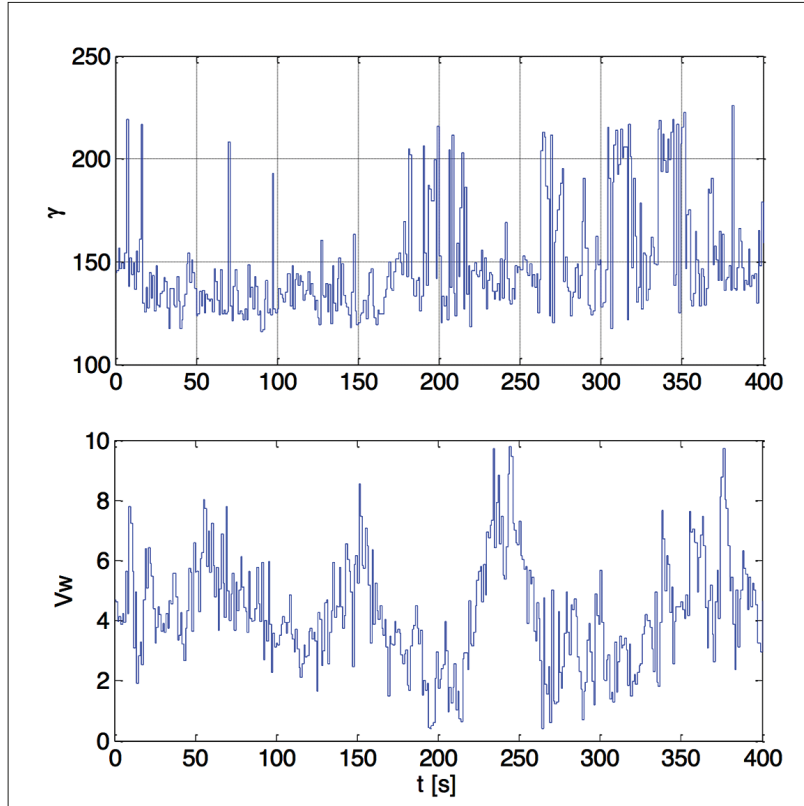

Fig. 19. Time series of the relative wind speed $V w[\mathrm{~m} / \mathrm{s}]$ and wind direction $\gamma[\mathrm{deg}]$

route will have sequential set rays, or the set rays will be determined successively based on knowledge of the ship's position and coordinates of the set waypoints. The SAR services prefer the first of these approaches, because the same disturbances also affect the missing object and the search should be carried out around a certain starting point, which will change its position when the same disturbances occur. However, if the search is to be carried out around a fixed reference point, then their simulated path is shown in Fig. 20.

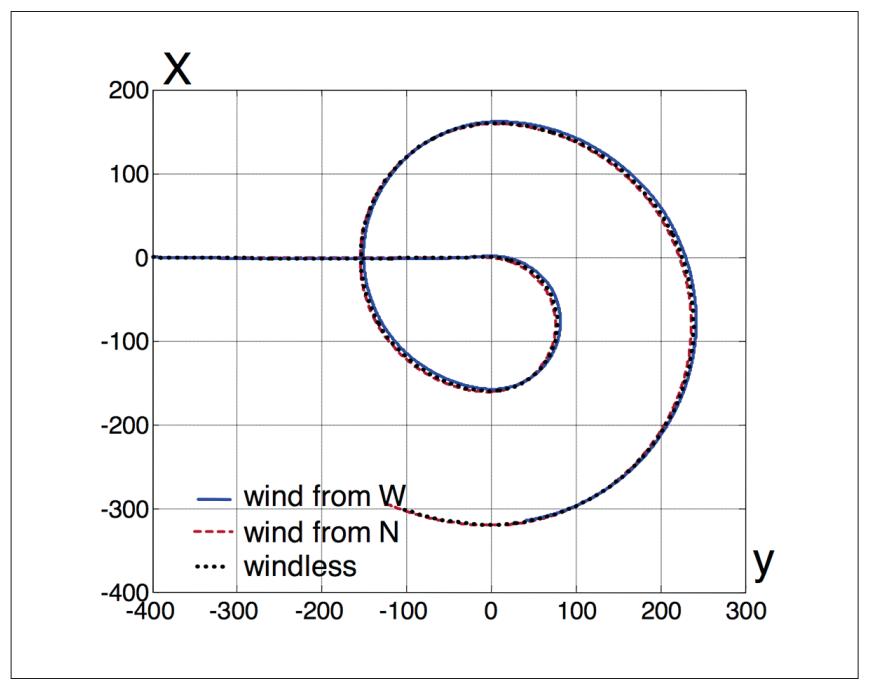

Fig. 20. Ship's trajectory during the search in the presence of north or west winds

\section{SUMMARY}

The presented innovative method of automatic ship guidance during the search for a missing object in a spiral is intended to shorten the time of sweeping the area around the starting point indicated by the SAR service. However, carrying out a rescue operation according to this pattern requires additional computer support. For its execution, a control system was designed using a technique for guiding the ship along a set trajectory in the shape of an arc. It allows a constant track spacing to be kept, which increases its reliability.

The spiral search process was checked and compared with the usual expanding square method by computer simulation of the movement of a surface unit with verified properties. The comparison was made on the basis of the detection probability and the previously defined performance indicators. The computer simulations confirmed that the open water area indicated by the SAR coordinator, determined by means of a circle with a given radius around the commence search point with the same main motor drive power, can be swept with a higher probability of success than would be the case after using the expanding square method. Simulation tests also showed that the proposed control system is capable of performing the test automatically and with high accuracy. Despite the promising results of the computer simulations, there is a need to conduct research on Lake Silm using a physical model to confirm the thesis. The spiral search method extends the time of wave action in sensitive sectors of its angle of attack, which may limit its application for smaller vessels without anti-roll stabilisers and therefore requires further research in this regard.

\section{ACKNOWLEDGEMENTS}

This research was supported by the Ministry of Science and Higher Education, within the framework of funds for research activity No. WE/2020/PZ/03.

\section{REFERENCES}

1. Ai Y., J. Lu, L.-L. Zhang L-L (2015): The optimization model for the location of maritime emergency supplies reserve bases and the configuration of salvage vessel. Transportation Research Part Vol. 83,170-188.

2. Azofra M., Pérez-Labajos C.A., Blanco B. (2007): Optimum placement of sea resources. Safety Sciencen Vol. 45 (9), 941-951.

3. Banda O.A.V. (2015): A Bayesian network for assessing the collision induced risk of an oil accident in the Gulf of Finland. Environmental Science \& Technology. 49, 5301-5309, doi: 10.1021/es501777g.

4. Burns R., Richter R. (1996): Neural-network approach to the control of surface ships. Control Eng. Practice, Vol. 4, No. 3, 411-416. 
5. Z. Burciu Z. (2010): Bayesian methods in reliability of search and rescue action. Polish Maritime Research. 4(67), Vol. 17, 72-78, 2010, doi: 10.2478/v10012-010-0039-76.

6. Goerlandt F, Torabihaghighi, Kujala P.(2013): A model for evaluating performance and reliability of the voluntary maritime rescue system in the Gulf of Finland. 11th International Conference Probabilistic Safety, Reliability and Risk Analysis: Beyond the Horizon, Amsterdam, Netherlands. 2013, 1351-1356.

7. Guoxiang L., Maofeng L., (2010): SARGIS: a GIS-based decision-making support system for maritime search and rescue, International Conference on E-Business and E-Government, doi: 10.1109/ICEE.2010.398.

8. H. N. Esfahani H.N., Szlapczynski R. (2019): Model Predictive Super-Twisting Sliding Mode Control for an Autonomous Surface Vehicle. Polish Maritime Research. No. 3, Vol. 26, 163-171, doi: 10.2478/pomr-2019-0057.

9. Fang M.-C., Luo J.-H. (2007): On the track keeping and roll reduction of the ship in random waves using different sliding mode controllers. Ocean Engineering 34, 479-488.

10. Fossen T.I. (2002): Marine control systems: guidance, navigation and control of ships, rigs and underwater vehicles. Marine Cybernetics. Trondheim. ISBN 82-92356-00-2.

11. Frost J.R., Cooper D.C., Robe Q. (2003): Compatibility of Land SAR Procedures with Search Theory. U.S. Department of Homeland Security United States Coast Guard Operations (G-OPR) Washington, D.C. 20593-0001.

12. Frost J.R.,. Stone L.D. (2001): Review of search theory: Advances and applications to search and rescue decision support. US Coast Guard Research and Development Center, Groton. 2001.

13. Koopman B.O. (1946): Search and screening. OEG Report No. 56, The Summary Reports Group of the Columbia University Division of War Research, Alexandria, Virginia, Center for Naval Analyses. 1946.

14. 14. Koopman B.O. (1980): Search and screening: general principles with historical applications. Revised. New York, Pergamon Press.

15. Kula K.S. (2015): Autopilot Using the Nonlinear Inverse Ship Model. Marine Navigation and Safety of Sea Transportation, CRC Press, ISBN: 978-1-138-02858-6, 101-108.

16. Kula K.S.,Tomera M. (2017): Control system of training ship keeping the desired path consisting of straight-lines and circular arcs. TransNav Journal Vol. 11 No. 4. 2017, 711-719, doi: 10.12716/1001.11.04.19.
17. IAMSAR Manual, IMO/ICAO-International Aeronautical and Maritime Search and Rescue Manual, Vol. III, Mission Co-ordination, London/Montreal. 2008.

18. Li L. (2006): Rescue vessel location modeling. MSc thesis. Halifax, Canada, Dalhousie University, Department of Industrial Engineering.

19. Liu Y., Bu R., Gao X. (2018): Ship Trajectory Tracking Control System Design Based on Sliding Mode Control Algorithm, Polish Maritime Research. No 3,Vol. 25, 26-34, doi: 10.2478/ pomr-2018-0093.

20. Morari M., Zafiriou E (1989): Robust process control. Englewood Cliffs, NJ Prentice Hall, 1989, ISBN-13:978-0137821532.

21. Nomoto K., Taguchi T., Honda K., Hirano S. (1957): On the steering qualities of ships. International Shipbuilding Progress 4, 354-370.

22. Norrington L., Quigley J., Russel A., Van der Meer R. (2008): Modeling the reliability of search and rescue operations with Bayesian Networks. Reliability Engineering \& System Safety Vol. 93(7), 940-949.

23. Richter R., Burns R. (1993): An artificial neural network autopilot for small vessels. Proceedings of the 1 st Conference of the UK Simulation Society, Edinburgh, 168-172.

24. Stone L.D.(1989): Theory of optimal search. Military Applications Section. Operations Research Society of America, Arlington, Virginia, ORSA Books.

25. Velasco F.J., López E.(2000): Predictive Control of Ship Steering Autopilots. $2^{\text {nd }}$ International Congress on Maritime Technological Innovations and Research, Spain, 89-98.

26. Witkowska A., R. Śmierzchalski (2012): Designing a ship course controller by applying the adaptive backstepping method. Int. J. Appl. Math. Comput. Sci., Vol. 22, No. 4, 985-997, doi: 10.2478/v10006-012-0073-y.

27. Tomera M. (2018): Switching-Based Multi-Operational Control of Ship Motion. Akademicka Oficyna Wydawnicza EXIT. Warszawa.

28. Tzeng. C.-Y. (1999): An internal model control approach to the design of yaw-rate-control ship-steering autopilot, IEEE Journal of Oceanic Engineering, Vol. 24, No. 4, pp. 507-513.

29. Yang Y.S., Ren J.S. (2003): Adaptive fuzzy robust tracking controller design via small gain approach and its application. IEEE Transactions on Fuzzy Systems, 11, 783-795.

30. Zeid I., Frost J.R. (2005) A decision support system for Canadian search and rescue operations', European Journal of Operation Research. 162 3, 630-653. 
31. Zwierzewicz Z. (2015): The design of ship autopilot by applying observer-based feedback linearization. Polish Maritime Research 22(1), 16-21.

\section{CONTACT WITH THE AUTHOR}

\section{Krzysztof Stefan Kula}

e-mail:k.kula@we.umg.edu.p

Gdynia Maritime University

Department of Ship Automation

Morska 83-87

81-225 Gdynia

Poland 